new one. A robot represents nothing more than a mechanical technician - it reproduces all the successes but also all the errors, so you don't move forward in your science."

The company produced a high-throughput system that needs no specialized knowledge to operate and can be programmed to carry out up to four common purification steps starting with affinity purification. "The skill of chromatography is sitting within the system," says Simpson. The software is written as a series of wizards, each representing a single step. GE will shortly be launching a software package for the purification of monoclonal antibodies.

The basic four-module set-up can purify up to 2,500 proteins a year, each module producing up to $50 \mathrm{mg}$ of protein per run. A twin-pack version is aimed at smaller labs wanting to purify up to 1,000 proteins a year.

The new protein-purification facility at Monash University in Melbourne, Australia, is deploying a 12-module ÄKTAxpress setup for its ambitious development programme. "I'm a structural biologist, so my interest is in producing large amounts of recombinant protein for structural and functional studies," says James Whisstock, scientific director of the facility. "What's really important is that the cost of equipment is within reach of a normal university laboratory set-up. There are some very big structural biology institutes with between US\$50 million and \$100 million's worth of industrial-scale protein preparation equipment. From our point of view that's not achievable, but we're bringing in this technology, which is going to make a huge difference to our research."

The ability to deal with many more proteins simultaneously will allow the lab to approach problems differently. "If you have a very challenging protein target and want to try 50 different constructs, at the moment it's really not feasible to do that manually one after the other," Whisstock points out. "Now, you can try the same molecule from 50 different species. With parallel advances in expression technology, the whole process is simplified and really streamlined, and provides the capacity to perform that experiment. You're trying so many different things simultaneously, you're likely to get a result."

\section{Automating innovation}

Several companies are developing equipment and product ranges that can be used at different points in the proteomics pipeline, from raw cell extracts to mass spectrometry and beyond. Beckman Coulter in Fullerton, California, is rolling out its ProteomeLab family to help with everything from initial purification of cell extracts, through protein fractionation and characterization, to the ultimate steps of disease diagnosis.

"We try to link technologies together to simplify the job for what takes place at the end, which is typically mass spectrometry," says John Hobbs, group product manager for ProteomeLab. "To get to that point, a lot of people have realized that it's garbage in, garbage out. If you put crap into a mass spectrometer,

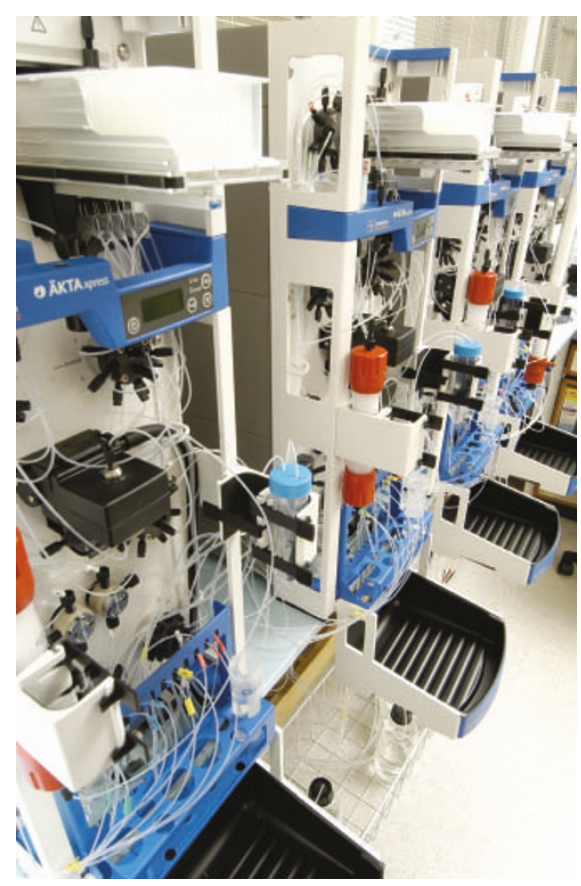

Scaling-up: ÄKTAxpress at Monash University's protein purification facility.

the results you get out will be the same."

The ProteomeLab PF 2D Protein Fractionation System automates two-dimensional chromatographic fractionation, resolving proteins by isoelectric point and hydrophobicity. The emphasis is on standardizing the protocols and techniques used by researchers. "Biologists want to be able to look at their results and see if they relate to someone else's,"

\title{
ATTRACTING ATTENTION
}

Magnetic beads have been used for protein separation since the 1980s, but the technology is now being adapted for new proteomic applications and use with automated platforms. The market leader in paramagnetic beads is Dynal Biotech based in Oslo, Norway, and recently acquired by life-sciences giant Invitrogen in Carlsbad, California.

Dynal has just signed a co-marketing agreement for its Dynabead kits and Tecan's Freedom EVO automated platform, and has developed protocols for other platforms such as Beckman Coulter's Biomek FX and the KingFisher magnetic separation platform from Thermo Electron of Waltham, Massachusetts. "The main purpose of having magnetic beads is that you can automate the whole process," says Lars Korsnes, director of research and development at Dynal. "The bead technology has some advantages compared with standard chromatography systems - while it's not so easy to put a whole-blood sample into a chromatography column, with magnetic beads you can put the whole sample in."

Dynabeads, like those from some other

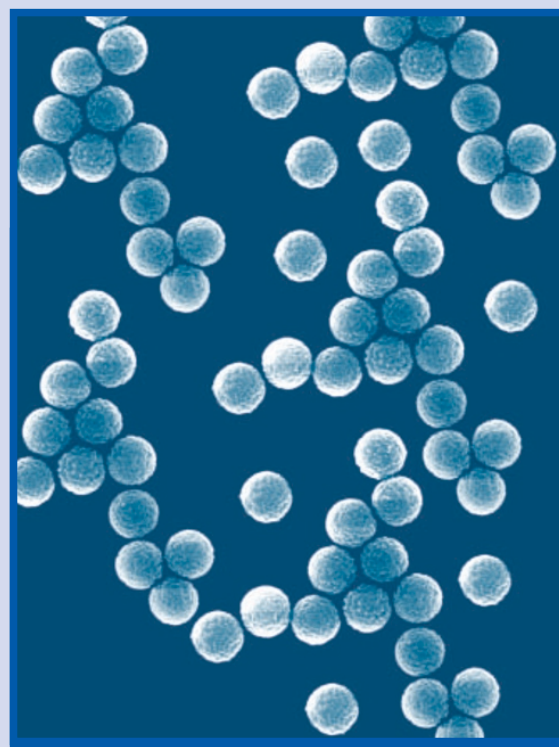

Magnetic beads are branching out. suppliers, are superparamagnetic, with no residual magnetism outside an applied magnetic field. They are also uniform in size, shape and surface properties. This all helps to prevent the beads clogging up an automated device, Korsnes notes.

Bead technology is also more scaleable than chromatography columns, although Dynal is concentrating on more analytical or small-scale protein isolation and protein fractionation for different applications in proteomics. The firm is currently launching a new range of beads with functionalities such as ion-exchange groups, reverse-phase chromatography and hydrophobic chemistries. New owner Invitrogen plans to apply Dynal's surface technologies to a wider range of products.

The beads are also showing promise in the challenging separation of membrane proteins. "There have been some publications where one can bind membrane proteins either before or after lysing the cells," Korsnes says. "Whether we will develop a special protocol is a question for the future, but the technology is there already." 